\title{
Two-dimensional Deterministic Cellular Automaton Traffic FI Model
}

\author{
Feng-Wei Yang ${ }^{1, a}$, Yang-Jie Wang ${ }^{1, b}$, Yan Guo ${ }^{1, c}$, Yu Xue ${ }^{1,2, d, *}$ \\ ${ }^{1}$ Institute of Physical Science and Engineering, Guangxi University, Nanning, Guangxi, 530004,
}

${ }^{2}$ The Key Laboratory of relativistic astrophysics, Guangxi, Nanning, Guangxi, 530004, China

a894736760@qq.com, bwuliwangyj@163.com, c2292603807@qq.com

${ }^{*}$ Corresponding author

Keywords: Cellular Automata, Traffic flow, FI model, Traffic jamming.

\begin{abstract}
Based on cellular automaton traffic BML model, one-dimensional traffic Fukui-Ishibashi(FI)model is extended to urban traffic network. The two-dimensional FI model is proposed. Under the conditions of periodic boundary, the relationship of the average speed and the density of the traffic as well as the fundamental diagram of the traffic for size of traffic network and the maximum speed of the vehicle are obtained by simulation. The result shows that the maximum speed has a great impact on traffic congestion under different size of traffic networks, and reveals transition from free flow to local jamming and fully jamming with the increase of density. The transition point to induce traffic congestion is decreased when the maximum velocity increases in the same lattice. The corresponding patterns of traffic in several states confirm these research results further.
\end{abstract}

\section{Introduction}

The rapid growth of social economy depends on the highly developed transportation. At the same time, the society and environment have seriou sly affected by the traffic jamming problems. Therefore, more and more scientists or researchers in different fields have been devoting to study the traffic flow since the last century 50s. Many complicated phenomena observed in the real traffic jamming have aroused a lot of people's great attentions [1]. To study all kinds of phenomena caused by the traffic jams, methods of modeling and computer simulation are used in recent years[2][3]. For the whole city, the simulation of the traffic flow is a very formidable task due to including a lot of complex factors such as the road conditions, intersection and so on[4].

The system of traffic flow is an asymmetric exclusion system and many traffic flow models are put forward [5]. One of the most famous traffic models is the cellular automata model (CA model), and the NaSch model is a typical one-dimensional CA model of traffic flow, which was put forward by Nagel and Schreckenberg in 1992[6]. Due to its simple modelling and easy parallel calculation, the cellular automaton traffic model is very good to reproduce the actual traffic phenomena and effectively simulate the movement of vehicles in traffic as well as describe all kinds of traffic factors [1-3]. On the basis of the NaSch model, many improved traffic flow models Such as T-STCA model, TT and FI model, etc. are proposed[1-3,7,11]. For one dimensional FI model, the vehicle speed of the next step was determined through headway of vehicle ahead, and the analytical solution of one dimensional FI model was derived[12], and its energy dissipation of traffic flow also can be discussed[13]. For the traffic network in urban, O.Biham, A.A.Middleton and D.Levine first put forward the two-dimensional cellular automaton traffic model called BML model in 1992. The urban traffic network is described with a two dimensional square lattice with size $\mathrm{L} \times \mathrm{L}$. The vehicles along two different directions are stochastically distributed at initial time, and then evolve according to the evolution of simple rules. The traffic congestion in urban can be simulated. In the year of 1993, Cuesta [5] et al. have proposed the improved BML model via introducing turn of the vehicle. Pecr[8] et al. firstly found that driver violating the traffic rules is the important reason of causing congestion in the urban traffic network by the introduction of game in BML model. In more recent years, Ding[9] et al. studied that the random update rule on the BML model has influence on traffic jamming, and presented the corresponding average field analytical solution. Xie[10] et al. studied the relationship of 
the speed and the density in the BML model by using the method of local traffic information for dynamic control. Kuang[14] et al. studied the effect of slow start has influence on the extend BML model with four directions of traffic flow.

In this paper, the two-dimensional FI model by extending on-dimensional FI model on traffic network is proposed based on the idea of BML model. We attempt to obtain the relationship of the average speed, the density and the flow of traffic and discuss congestion problem on the traffic network by simulation.

\section{Two-Dimensional CA FI Model}

In a two-dimensional cellular automaton BML model, the urban traffic network was described with a two-dimensional square lattice of size $\mathrm{L} \times \mathrm{L}$. $\mathrm{L}$ is the length of each side on square lattice. There are two kinds of vehicle in the BML model, those who can only move advance towards north or east respectively. Two types of vehicles are uniformly distributed with equal probability $\rho / 2$ on a two-dimensional periodic square lattice. Since there are traffic lights on every intersection, eastbound and northbound vehicles take turns to move one step forward. Normally, eastbound vehicle are arranged to move on even steps. Thus, state of each site is dominated by an eastbound, northbound vehicle and empty. Based on BML model, a one-dimensional cellular automata FI model is extended to the one in two-dimensional discrete lattice. A site may be occupied by a vehicle with the maximum $\mathrm{V} m$ or not at a certain moment. A vehicle in the position $(\mathrm{x}, \mathrm{y})$ at time $\mathrm{t}$ is represented by $\mathrm{M}(\mathrm{x}, \mathrm{y}, \mathrm{t})$. The eastbound vehicle moves towards the right direction in every odd time step. When there is a vehicle to occupy a grid in the nearest neighbor coordinate of $\left(\mathrm{x}_{1}, \mathrm{y}_{1}\right)$ in front of it, the gap between them is gap $=M\left(x_{1}, y_{1}, t\right)-M(x, y, t)$. Analogously, a northbound vehicle moves towards the north direction in every even time step. The gap of its the nearest neighbor vehicle in front is gap $=\mathrm{M}\left(\mathrm{x}_{2}, \mathrm{y}_{2}, \mathrm{t}\right)-\mathrm{M}(\mathrm{x}, \mathrm{y}, \mathrm{t})$. Then all of the vehicles update its state according to the following evolution rule similarity to FI model.

1)The update speed of all vehicles in $t+1$ time step:

If $\mathrm{Vm} \leq$ gap, then

else

$$
\mathrm{v}(\mathrm{x}, \mathrm{y}, \mathrm{t}+1)=\left\{\begin{array}{lr}
\mathrm{Vm}, & \text { probability } 1-\mathrm{p} ; \\
\mathrm{Vm}-1, & \text { probability } \mathrm{p} .
\end{array}\right.
$$

$$
\text { If } \mathrm{Vm}>\text { gap, so } \quad \mathrm{v}(\mathrm{x}, \mathrm{y}, \mathrm{t}+1)=\text { gap. }
$$

2) The update position of all vehicles in $\mathrm{t}+1$ time step:

$$
\mathrm{M}(x, y, t+1)=M\left(x_{0}+y_{0}+t\right)+v(x, y, t)
$$

Where $\mathrm{Vm}$ is the maximum speed of the vehicle be allowed to move. Every vehicle speed can be in the range of 0 and $\mathrm{Vm}$, and $\mathrm{p}$ is delay probability.

At the beginning of the computer simulation, the vehicles are distributed at random on square lattice with size $\mathrm{L} \times \mathrm{L}$. The total density of the vehicles is $\rho=\left(\mathrm{n}_{\rightarrow}+\mathrm{n}_{\uparrow}\right) /\left(\mathrm{L}_{x} \times \mathrm{L}_{y}\right)$, the $\mathrm{n}_{\rightarrow}$ and $\mathrm{n}_{\uparrow}$ is the number of the eastbound, northbound vehicle vehicles, repectively. The density of the eastbound (northbound) vehicles is $\rho_{x}\left(\rho_{\mathrm{y}}\right)=\mathrm{n}_{\rightarrow}\left(\mathrm{n}_{\uparrow}\right) /\left(\mathrm{L}_{x} \times \mathrm{L}_{y}\right)$. The speed of each vehicle is determined by the evolutional rules in every time step.The average speed $\mathrm{V}$ of vehicle is calculated by the two odd and even time step: $V=\sum_{i} v_{\rightarrow}^{i} / n_{\rightarrow}+\sum_{i} v_{\uparrow}^{i} / n_{\uparrow}, v_{\rightarrow}^{i}\left(v_{\uparrow}^{i}\right)$ is the speed of the i vehicle in any time step. To study the traffic jamming on the network, it can dimensionlessly simulate under the size of network $\mathrm{L}_{\mathrm{x}} \times \mathrm{L}_{\mathrm{y}}=256 \times 256$ as well as the different maximum speed $\mathrm{Vm}=1,3,5,7$ and 9 using the deterministic CA FI model with the probability $\mathrm{p}=0.0$. The simulation is carried out after the relaxation time $\mathrm{Tc}=10000$ and the total simulation time is $\mathrm{T}=2 \times 104$. Each average quantity is obtained via 30 running, and the periodic boundary condition is adopted.

\section{Simulation And Analysis}

Fig. 1(a) shows that the relationship of the average speed $v$ and the density $p$ in the different maximum speed of vehicle $\mathrm{V}_{\mathrm{m}}=1,3,5,7$ and 9. From Fig.1(a), it can see that the average speed of 
vehicle on traffic network is equal to the maximum allowed speed of vehicle in small density. For example, when the maximum allowed speed of vehicle is $\mathrm{V}_{\mathrm{m}}=9$ and density is less than 0.075 , the average speed of vehicle on traffic network is $V_{m}=9$. That is to say, traffic of low-density on network is not in congestion. There is no traffic jam in interval of the density $\rho \varepsilon[0.0,0.30]$ when the maximum allowed speed of vehicle is $\mathrm{V}_{\mathrm{m}}=1$, which is similar to the model in [9]. When the density exceeds 0.3 and the average $\mathrm{V}$ of vehicle decrease, it implies that vehicle starts to reduce its velocity due to other vehicle in front. For the maximum allowed speed of vehicle $\mathrm{V}_{\mathrm{m}}=9$, vehicles reduce their velocity in the range of the density $\rho=0.075$ and $\rho=0.15$. That is to say, local traffic congestion occurs, which can be illustrated by the pattern of traffic in Fig.2(a). For other maximum allowed speed of vehicle like $V_{m}=3,5$ and 7, the trend of local traffic congestion is same in the middle density of vehicle. Finally, the traffic on network trends to fully jamming in high density. Fig.1(b) shows that the relationship of the flow and the density $\rho$ in the different maximum allowed speeds. When the density $\rho$ increases to a certain value, the flow of traffic begins to increase and reach the peak. When the density exceeds the certain critical value, the flow is decreased with the increasing of density, and finally trends to the zero.

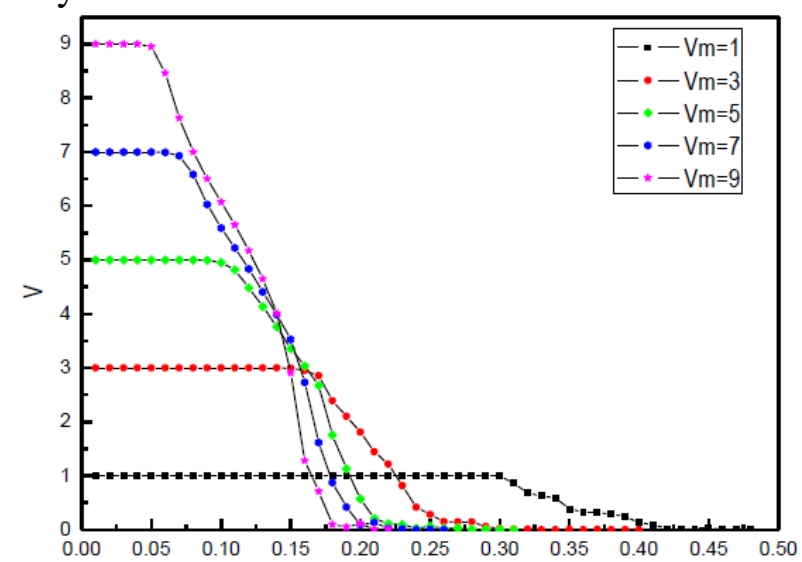

(a)

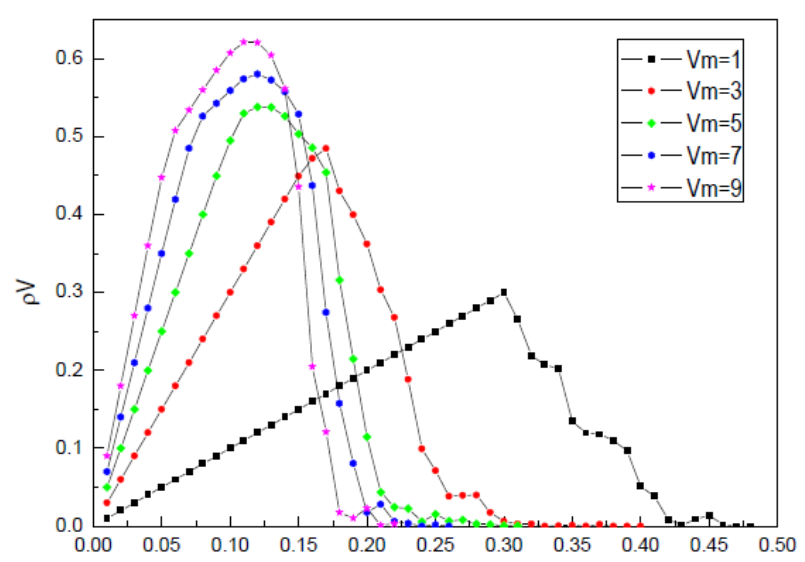

(b)

Fig.1(a) the relationship of the average speed $\mathrm{v}$ and the density $\rho$ in different maximum speed of vehicle. (b) flow against density $\rho$ in different maximum speed of vehicle

The evolution processes of traffic on network can be reflected in the several traffic patterns. Fig. 3(a),(b) and (c) show the corresponding to the traffic pattern in small, middle and high density for the maximum allowed speed of vehicle $\mathrm{V}_{\mathrm{m}}=9$. When the density $\rho=0.01$, Fig.2(a) clearly reveals no jam. When the density $\rho$ is 0.10 , local traffic jamming emerges and Fig.2(b) shows local clustering of standstill vehicles. When the density $\rho=0.20$, full congestion of traffic is exhibited along the diagonal line from the left bottom to the upper right corner.

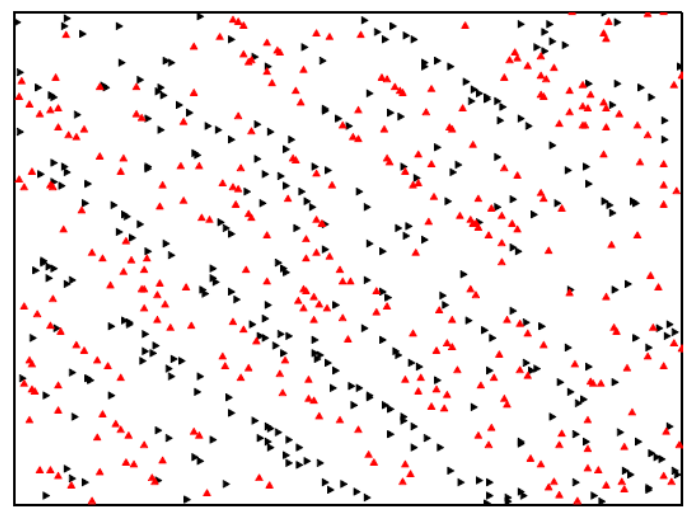

(a) $\rho=0.01$

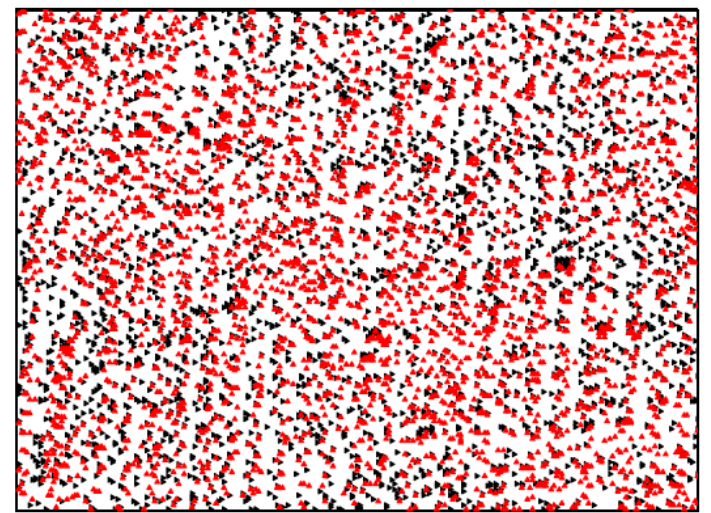

(b) $\rho=0.10$ 


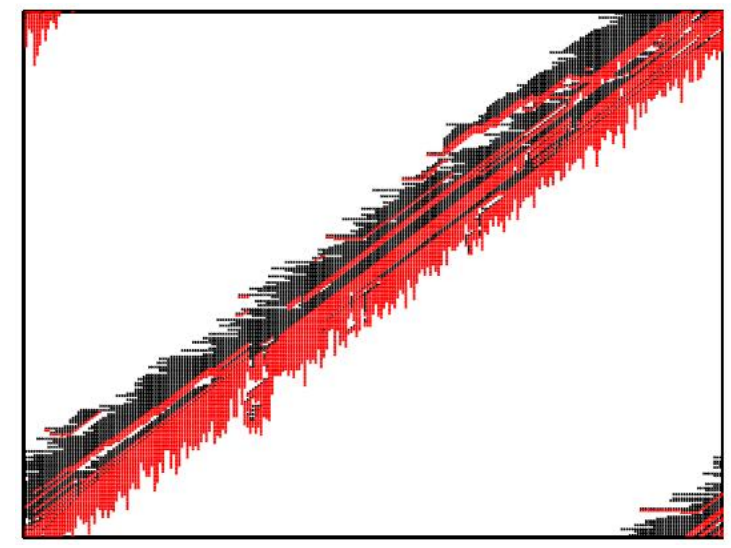

(c) $\rho=0.20$

Fig. 2 traffic pattern in the density of vehicle $\rho=0.01,0.10$ and 0.20 . The black arrow denotes the eastbound vehicle and the red arrow is northbound one

\section{Conclusion}

In this paper, the deterministic two-dimensional cellular automata FI model is proposed based on the BML model of traffic network. The influences of the different flow and the maximum speed on the traffic jam are studied by numerical simulation under the periodic boundary conditions. The result shows that the maximum speed has a great impact on traffic congestion under different size of traffic networks, and reveals transition from free flow to local jamming and fully jamming with the increase of density. Under the condition of the identical lattice, the transition point to cause traffic congestion is decreased when the maximum velocity increases. The corresponding patterns of traffic in several states confirm these research results further.

\section{Acknowledgement}

This paper is supported by the National Natural Science Foundation of China (Grant Nos. 11262003), Undergraduate experiment skill, Scientific and Technological Innovation Project of Guangxi University (No. SYJN 20130415), Scientific Research Fund of Guangxi Education Department ( No.2013ZD059).

\section{References}

[1] Chowdhury D, Santen L, Schadschneider A,and Schreckenberg M., "Statistical physics of vehicular traffic and some related systems.” Phys Rep, vol. 329 (2000) pp.199-329.

[2] Helbing D.,“Traffic and related self-driven many-particle systems.”Rev Mod Phys, vol.73 (2001) pp.1067 -1141.

[3] Maerivoet S, DeMoor B. "Cellular automata models of road traffic.”Phys Rep, vol. 419 (2005) pp $1-64$.

[4] O. Biham, A. A. Middleton, D. Levine,"Self organization and a dynamical transition in traffic flow models" Phys.Rev.A. vol.46 (1992) pp. 6124-6127.

[5] Cuesta J A, Matínez F C, Molera J M and Sánchez A, "Phase transitions in two-dimensional traffic-flow models”Phys. Rev. E, vol. 48 (1993) pp.R4175-4179.

[6]K.Nagel,M.J.Schreckenberg,“A cellular automaton model for freeway traffic“" J.Phys.I,vol.2 (1992) pp.2221-2233

[7] Takayasu M, Takayasu H,“1/f noise in a traffic model”Fractals, vol.1 (1993) pp. 860-866. 
[8] Perc M "Premature seizure of traffic flow due to the introduction of evolutionary games" New J. Phys. Vol.9 (2007) pp. 10003-10020.

[9] Ding Z J, Jiang R and Wang B H,"Traffic flow in the Biham-Middleton- Levine model with random update rule”Phys. Rev. E,vol. 83 (2011) pp. 047101-047109.

[10] Xie J R, Jiang R, Ding Z J, Li Q L and Wang B H,"Dynamical traffic light strategy in the Biham-Middleton-Levine model”Phys. Rev. E, vol.87 (2013) pp. 022812-022819.

[11] M.Fukui, Y.Ishibashi," "traffic flow in 1D cellular automaton model includeing cars moving with high speed”vol.68 (1996) pp. 1868-1870.

[12] Wang B.H., L.Wang,P.M.Hui and B.H, STAT PHYS 20 The 20th IUPAP International Conference on Statistical Physics,(Paris,July 20-24,1998) Oral contribution:Short Communications.

[13] Wen Jian, Tian H H, Kang S J, Xue Y, "Study on the Energy Consumption of the Cellular Automaton FI Model for Mixed Traffic Flow”, vol.59 (2010) pp. 7693-7699.

[14] Kuang H, Zhang G X, Li X L and Lo S M, Effect of slow-to-start in the extended BML model with four-direction traffic, Phys. Lett. A, 378 (2014) 1455-1460. 